\title{
$h$-PRINCIPLES FOR CURVES AND KNOTS OF CONSTANT CURVATURE
}

\author{
MOHAMMAD GHOMI
}

\begin{abstract}
We prove that $\mathcal{C}^{\infty}$ curves of constant curvature satisfy, in the sense of Gromov, the relative $\mathcal{C}^{1}$-dense $h$-principle in the space of immersed curves in Euclidean space $\mathbf{R}^{n \geq 3}$. In particular, in the isotopy class of any given $\mathcal{C}^{1}$ knot $f$ there exists a $\mathcal{C}^{\infty}$ knot $\tilde{f}$ of constant curvature which is $\mathcal{C}^{1}$-close to $f$. More importantly, we show that if $f$ is $\mathcal{C}^{2}$, then the curvature of $\tilde{f}$ may be set equal to any constant $c$ which is not smaller than the maximum curvature of $f$. We may also require that $\widetilde{f}$ be tangent to $f$ along any finite set of prescribed points, and coincide with $f$ over any compact set with an open neighborhood where $f$ has constant curvature $c$. The proof involves some basic convexity theory, and a sharp estimate for the position of the average value of a parameterized curve within its convex hull.
\end{abstract}

\section{INTRODUCTION}

In this work we show that any smooth $\left(\mathcal{C}^{\infty}\right)$ curve immersed in Euclidean space $\mathbf{R}^{n \geq 3}$, may be deformed to one with constant curvature by an arbitrarily small perturbation of the curve and its tangent lines. Thus we may say that there exists no "visual difference" between an arbitrary smooth curve and a curve whose curvature is constant. Further we show that this constant may take on any value not smaller than the maximum of the curvature of the initial curve, which, as we verify in the appendix, is the optimal lower bound.

To state our main result precisely, we let $\Gamma$ stand for either an interval $[a, b] \subset \mathbf{R}$, or a topological circle $\mathbf{R} /(b-a) \mathbf{Z}$, and let $\mathcal{C}^{\alpha}\left(\Gamma, \mathbf{R}^{n}\right)$ be the space of maps, or curves, $f: \Gamma \rightarrow \mathbf{R}^{n}$ whose derivatives $f^{(i)}$ exist and are continuous for $0 \leq i \leq \alpha$. The standard norm on $\mathcal{C}^{\alpha}\left(\Gamma, \mathbf{R}^{n}\right)$ is denoted by

$$
\|f\|_{\alpha}:=\sup \left\{\left|f_{j}^{(i)}(t)\right| \mid t \in \Gamma, 1 \leq i \leq \alpha, 1 \leq j \leq n\right\},
$$

where $f_{j}$ are component functions of $f$. For $\alpha \geq 1$, the space of immersions $\operatorname{Imm}^{\alpha}\left(\Gamma, \mathbf{R}^{n}\right) \subset \mathcal{C}^{\alpha}\left(\Gamma, \mathbf{R}^{n}\right)$ consists of those curves with nonvanishing speed $\left\|f^{\prime}\right\|$. We say that $f_{0}, f_{1} \in \operatorname{Imm}^{1}\left(\Gamma, \mathbf{R}^{n}\right)$ are regularly homotopic if there exists a one parameter family of curves $f_{t} \in \operatorname{Imm}^{1}\left(\Gamma, \mathbf{R}^{n}\right), t \in[0,1]$, such that $t \mapsto f_{t}$ is continuous with respect to the $\mathcal{C}^{1}$-norm $\|\cdot\|_{1}$. Then $f_{t}$ is called a regular homotopy between $f_{0}$ and $f_{1}$. If $\alpha \geq 2$, the curvature of $f \in \operatorname{Imm}^{\alpha}\left(\Gamma, \mathbf{R}^{n}\right)$ is defined as

$$
\kappa:=\frac{\left\|T^{\prime}\right\|}{\left\|f^{\prime}\right\|}, \quad \text { where } \quad T:=\frac{f^{\prime}}{\left\|f^{\prime}\right\|}
$$

Date: Last revised on June 28, 2007.

2000 Mathematics Subject Classification. Primary 53A04, 55A25; Secondary 58C35, 53C21.

Key words and phrases. h-principle, knot, constant curvature, convex integration.

Supported in part by NSF CAREER award DMS-0332333. 
is the tantrix of $f$. The space of embeddings $\operatorname{Emb}^{\alpha}\left(\Gamma, \mathbf{R}^{n}\right) \subset \operatorname{Imm}^{\alpha}\left(\Gamma, \mathbf{R}^{n}\right)$ is the collection of those curves which are one-to-one. A pair of embeddings $f_{0}, f_{1} \in$ $\operatorname{Emb}\left(\Gamma, \mathbf{R}^{n}\right)$ are (regularly) isotopic if they can be joined by a regular homotopy of embeddings $f_{t} \in \operatorname{Emb}\left(\Gamma, \mathbf{R}^{n}\right)$.

Theorem 1.1. Let $f_{0} \in \operatorname{Imm}^{\alpha \geq 2}\left(\Gamma, \mathbf{R}^{n \geq 3}\right)$ be an immersed curve with curvature $\kappa_{0}$. Then for any $\epsilon>0$, and $c \geq \max \left[\kappa_{0}\right]$, there exists a regular homotopy $f_{t} \epsilon$ $\operatorname{Imm}^{\alpha}\left(\Gamma, \mathbf{R}^{n}\right), t \in[0,1]$, such that $\left\|f_{0}-f_{t}\right\|_{1} \leq \epsilon$ for all $t$, and $f_{1}$ has constant curvature c. If $f_{0}$ is an embedding, then (choosing $\epsilon$ sufficiently small) we may require that $f_{t}$ be an isotopy. Further, we may require that $f_{t}$ remain tangent to $f$ along any finite set of prescribed points of $\Gamma$. Furthermore, we may keep $f_{t}$ fixed on any compact subset of $\Gamma$ which has an open neighborhood where $\kappa_{0}=c$.

An embedding $f \in \operatorname{Emb}\left(\Gamma, \mathbf{R}^{n}\right)$ is a knot when $\Gamma$ is a circle. Since embeddings are open in $\mathcal{C}^{1}\left(\Gamma, \mathbf{R}^{n}\right)$, the above theorem implies that there exists a $\mathcal{C}^{\infty}$ knot of constant curvature in each isotopy class of knots. The first explicit construction for $\mathcal{C}^{2}$ knots of constant curvature are due to Koch and Engelhardt [8], who spliced together segments of helices; see also [9] where this method was used to obtain $\mathcal{C}^{2}$ knots in each isotopy class, and [2] where $\mathcal{C}^{\infty}$, even analytic, knots were constructed in some isotopy classes; however, these results do not include approximations of the type we discuss here, nor do they yield $\mathcal{C}^{\infty}$ knots in each isotopy class.

The methods we use here have the flavor of "convex integration" techniques which are used to prove some manifestations of the "h-principle". Indeed, in the terminology of Gromov [6], see also Eliashberg-Mishachev [1] or Spring [11], we show that curves of constant curvature satisfy the relative $\mathcal{C}^{1}$-dense $h$-principle in the space of $\mathcal{C}^{\alpha \geq 2}$ immersions. Yet we do not appeal to, nor are we aware of, any general $h$-principle theorems which may be immediately relevant; instead, we give a direct proof which is for the most part constructive.

The general outline for proving Theorem 1.1 is as follows. We may assume that the initial curve $f_{0}$ has unit speed. Then $\kappa_{0}=\left\|T_{0}^{\prime}\right\|$, i.e., the curvature of $f_{0}$ is the speed of its tantrix $T_{0}: \Gamma \rightarrow \mathbf{S}^{n-1}$. To find $f_{1}$ we use some basic convexity theory to construct a curve $T_{1}: \Gamma \rightarrow \mathbf{S}^{n-1}$ which (i) has constant speed, (ii) is $\mathcal{C}^{0}$-close to $T_{0}$, and most importantly (iii) has the same total integral (the first two conditions are fairly easy to achieve; however, the third condition requires more care). Then $f_{1}(t):=f_{0}(a)+\int_{a}^{t} T_{1}(s) d s$ has constant curvature, is $\mathcal{C}^{1}$ close to $f_{0}$, and coincides with $f_{0}$ on the end points of $\Gamma$. The desired homotopy is then given by the linear interpolation $f_{t}:=(1-t) f_{0}+t f_{1}$. To obtain the precise lower bound for the curvature of $f_{1}$ we need to show that the speed of $T_{1}$ can be set equal to any constant not smaller than the maximum speed of $T_{0}$. This requires a sharp estimate, which we obtain in Section 2 below, for the position of the average value of a curve in relation to its center of mass.

As we plan to show in a sequel to this work, the methods developed here may also be used to prove parametric versions of the $h$-principle for curves and knots of constant curvature. This would provide analogues to theorems of Feldman [3] and 
Gluck and Pan [5], see also [4], who studied, respectively, the regular homotopy and isotopy of closed curves with nonvanishing curvature.

\section{Averages and Centers of Mass of Curves}

For any set $X \subset \mathbf{R}^{n}$, let relint conv $X$ denote the relative interior of the convex hull of $X$. We recall that $p \in \operatorname{relint} \operatorname{conv} X$ if and only if $X$ lies on both sides of any hyperplane $H \subset \mathbf{R}^{n}$ which passes through $p$ and does not contain $X$. The average of a curve $f \in \mathcal{C}^{0}\left([a, b], \mathbf{R}^{n}\right)$ is defined as

$$
\text { ave }[f]:=\frac{1}{b-a} \int_{a}^{b} f(t) d t .
$$

Let relint conv $[f]$ denote the relative interior of the convex hull of $f([a, b])$.

Lemma 2.1. For any $f \in \mathcal{C}^{0}\left([a, b], \mathbf{R}^{n}\right)$, ave $[f] \in \operatorname{relint} \operatorname{conv}[f]$.

Proof. Let $u \in \mathbf{S}^{n-1}$, and set

$$
g(t):=\langle f(t)-\operatorname{ave}[f], u\rangle .
$$

Then $\int_{a}^{b} g(t) d t=0$. Thus, since $f$ and consequently $g$ is continuous, either $g$ vanishes everywhere or else it changes sign. Let

$$
H_{u}:=\left\{p \in \mathbf{R}^{n} \mid\langle p-\operatorname{ave}[f], u\rangle=0\right\}
$$

be the hyperplane passing through ave $[f]$, and orthogonal to $u$. Then it follows that either $f([a, b])$ lies entirely in $H_{u}$ or lies on both sides of $H_{u}$, which completes the proof.

For $f \in \mathcal{C}^{1}\left([a, b], \mathbf{R}^{n}\right)$ let $\mathrm{cm}[f]$ be the center of mass or geometric average of $f$, i.e.,

where

$$
\operatorname{cm}[f]:=\frac{1}{\operatorname{length}[f]} \int_{a}^{b} f(t)\left\|f^{\prime}(t)\right\| d t,
$$

$$
\text { length }[f]:=\int_{a}^{b}\left\|f^{\prime}(t)\right\| d t .
$$

Note that if $g:[c, d] \rightarrow \mathbf{R}^{n}$ is any reparameterization of $f$, i.e., there exists a diffeomorphism $\theta:[c, d] \rightarrow[a, b]$ such that $g=f \circ \theta$, then $\mathrm{cm}[g]=\mathrm{cm}[f]$. The following observation is also immediate:

$$
\text { ave }[f]=\operatorname{cm}[f], \quad \text { when } \quad\left\|f^{\prime}\right\|=\text { const. }
$$

The main aim of this section is to estimate the distance between the average of a curve and its center of mass (when the speed of the curve is not constant). To this end, for any (density) function $\rho \in \mathcal{C}^{0}([a, b], \mathbf{R})$ and $f \in \operatorname{Imm}^{1}\left([a, b], \mathbf{R}^{n}\right)$ we define

$$
\operatorname{cm}[f, \rho]:=\frac{\int_{a}^{b} f(t) \rho(t)\left\|f^{\prime}(t)\right\| d t}{\int_{a}^{b} \rho(t)\left\|f^{\prime}(t)\right\| d t} .
$$


Thus $\operatorname{cm}[f, \rho]=\operatorname{cm}[f]$ when $\rho \equiv 1$. Furthermore note that

$$
\operatorname{ave}[f]=\mathrm{cm}\left[f, \frac{1}{\left\|f^{\prime}\right\|}\right] \text {. }
$$

Conversely, we can show

Lemma 2.2. For any $f \in \operatorname{Imm}^{1}\left([a, b], \mathbf{R}^{n}\right)$ and $\rho \in \mathcal{C}^{0}([a, b], \mathbf{R})$ such that $\rho>0$ there exists a $\mathcal{C}^{1}$ diffeomorphism $\theta:[c, d] \rightarrow[a, b]$ such that

$$
\operatorname{cm}[f, \rho]=\operatorname{ave}[f \circ \theta] .
$$

Proof. Note that by (4) we have

$$
\operatorname{ave}[f \circ \theta]=\mathrm{cm}\left[f \circ \theta, \frac{1}{\left\|(f \circ \theta)^{\prime}\right\|}\right] .
$$

Further, since the center of mass is invariant under reparameterizations,

$$
\operatorname{cm}[f, \rho]=\operatorname{cm}[f \circ \theta, \rho \circ \theta],
$$

for any diffeomorphism $\theta:[c, d] \rightarrow[a, b]$. Thus, for the left hand side of the last two equations to be equal, we need to have

$$
\rho \circ \theta=\frac{1}{\left\|f^{\prime}(\theta) \cdot \theta^{\prime}\right\|},
$$

which is equivalent to

$$
\rho=\frac{1}{\left\|f^{\prime} \cdot \theta^{\prime}\left(\theta^{-1}\right)\right\|}=\frac{\left|\left(\theta^{-1}\right)^{\prime}\right|}{\left\|f^{\prime}\right\|} .
$$

Hence if we define (the mass function) $m:[a, b] \rightarrow \mathbf{R}$ by

$$
m(t):=\int_{a}^{t} \rho(u)\left\|f^{\prime}(u)\right\| d u,
$$

then $\theta:=m^{-1}:[0, m(b)] \rightarrow[a, b]$ is the desired mapping. In particular note that since by assumption $\rho$ is continuous and $\rho>0, m$ is $\mathcal{C}^{1}$ on $[a, b]$ and $m^{\prime}$ never vanishes. So $\theta$ is well-defined and is $\mathcal{C}^{1}$.

The last two lemmas may now be combined to obtain the following result. It gives conditions for the center of mass of a curve with nonconstant density to lie in the relative interior of the convex hull of the curve. We say $H$ is a proper support hyperplane of a set $X \subset \mathbf{R}^{n}$ provided that $X$ lies on one side of $H$, and intersects $H$ at some point, but is not contained entirely in $H$. The support of a function $f$, i.e., the closure of the set of points where $f \neq 0$, is denoted by $\operatorname{supp}[f]$.

Lemma 2.3. For any $f \in \operatorname{Imm}^{1}\left([a, b], \mathbf{R}^{n}\right)$ and $\rho \in \mathcal{C}^{0}([a, b], \mathbf{R})$ such that $\rho \geq 0$ and $\rho \neq \equiv$,

$$
\operatorname{cm}[f, \rho] \in \operatorname{conv}[f] .
$$

Furthermore, if there exists no proper support hyperplane of $f([a, b])$ which contains $f(\operatorname{supp}[\rho])$, then

$$
\operatorname{cm}[f, \rho] \in \operatorname{relint} \operatorname{conv}[f] .
$$


Note that the last condition in the above lemma is essential, for if there exists a proper support hyperplane $H$ of $f([a, b])$ which contains $f(\operatorname{supp}[\rho])$, then $\operatorname{cm}[f, \rho]$ lies in $H$ and therefore $\operatorname{cm}[f, \rho] \notin \operatorname{relint} \operatorname{conv}[f]$.

Proof. By Lemmas 2.1 and 2.2, $\operatorname{cm}[f, \rho+\epsilon] \in \operatorname{conv}[f]$ for any $\epsilon>0$. Thus since $\mathrm{cm}[f, \rho+\epsilon]$ converges to $\mathrm{cm}[f, \rho]$ as $\epsilon$ gets smaller, and conv $[f]$ is compact, (5) follows. Next note that we may assume int conv $[f] \neq \emptyset$, for otherwise we may reduce the dimension of $\mathbf{R}^{n}$, after an affine transformation. Let $H$ be a support hyperplane of $f([a, b])$. Then $f([a, b])$ cannot lie entirely in $H$. Consequently, by assumption $f(\operatorname{supp}[\rho])$ cannot lie entirely in $H$ either. Thus after a rigid motion we may assume that $H$ coincides with the hyperplane of the first $n-1$ coordinates in $\mathbf{R}^{n}$ and $f(\operatorname{supp}[\rho])$ lies above $H$. Then $\left\langle f(t) \rho(t), e_{n}\right\rangle$ is not identically zero, where $e_{n}:=(0,0, \ldots, 1)$. Thus $\left\langle\operatorname{cm}[f, \rho], e_{n}\right\rangle>0$. So $\mathrm{cm}[f, \rho]$ cannot lie in $H$. Since $H$ was chosen arbitrarily, we conclude that $\mathrm{cm}[f, \rho]$ cannot lie in any support hyperplane of $\operatorname{conv}[f]$ and therefore must be an interior point.

Finally we are ready to prove the main result of this section. Note that if $X \subset \mathbf{R}^{n}$ is any subset and $p \in X$, then, for any $\lambda \in[0,1]$,

$$
\lambda p+(1-\lambda) X:=\{\lambda p+(1-\lambda) x \mid x \in X\}
$$

gives the contraction of $X$ by a factor of $(1-\lambda)$ about $p$. The following result gives the smallest factor for the contraction of the convex hull of a curve about its center of mass to contain the average value of the curve.

Proposition 2.4. Let $f \in \operatorname{Imm}^{1}\left([a, b], \mathbf{R}^{n}\right)$. Then,

$$
\text { ave }[f] \in \lambda \mathrm{cm}[f]+(1-\lambda) \operatorname{conv}[f],
$$

for any constant

$$
0 \leq \lambda \leq \frac{\operatorname{ave}\left[\left\|f^{\prime}\right\|\right]}{\max \left[\left\|f^{\prime}\right\|\right]}
$$

Furthermore,

$$
\text { ave }[f] \in \lambda \mathrm{cm}[f]+(1-\lambda) \operatorname{relint} \operatorname{conv}[f]
$$

if either

$$
0 \leq \lambda<\frac{\text { ave }\left[\left\|f^{\prime}\right\|\right]}{\max \left[\left\|f^{\prime}\right\|\right]}
$$

or else, there exists no proper support hyperplane of $f([a, b])$ which contains $f\left(\operatorname{supp}\left[\max \left[\left\|f^{\prime}\right\|\right]-\left\|f^{\prime}\right\|\right]\right)$.

Proof. Note that if we define $\widetilde{f} \in \operatorname{Imm}^{1}\left([0,1], \mathbf{R}^{n}\right)$, by $\widetilde{f}(t):=f(a+(b-a) t) /(b-a)$, then $\tilde{f}^{\prime}=f^{\prime}$, while ave $[\widetilde{f}]=$ ave $[f] /(b-a), \operatorname{cm}[\widetilde{f}]=\operatorname{cm}[f] /(b-a)$, and $\operatorname{conv}[\widetilde{f}]=$ $\operatorname{conv}[f] /(b-a)$. Thus $(7)$ holds for $f$ if and only if they holds for $\tilde{f}$. So, for convenience, we may assume that $a=0$ and $b=1$. Further, after a dilation of $\mathbf{R}^{n}$ we may assume that length $[f]=1$, which forces ave $\left\|f^{\prime}\right\|=1$. Also note that if $(7)$ holds for some $f$, then it holds for $f+p$ for any $p \in \mathbf{R}^{n}$. Thus we may assume that

$$
\mathrm{cm}[f]=o,
$$


the origin of $\mathbf{R}^{n}$. Then we just need to verify that

$$
\text { ave }[f] \in(1-\lambda) \operatorname{conv}[f] \text {. }
$$

for all $f \in \operatorname{Imm}^{1}\left([0,1], \mathbf{R}^{n}\right)$ with $\operatorname{cm}[f]=o$ and length $[f]=1$, and all $0 \leq \lambda \leq$ $1 / \max \left[\left\|f^{\prime}\right\|\right]$. To this end recall that ave $[f]=\operatorname{cm}[f, \rho]$, where $\rho:=1 /\left\|f^{\prime}\right\|$. Thus the above relation may be rewritten as

$$
\operatorname{cm}[f, \rho] \in(1-\lambda) \operatorname{conv}[f] .
$$

If $\lambda=1$, then $\left\|f^{\prime}\right\|$ is constant, in which case $\operatorname{cm}[f, \rho]=\operatorname{cm}[f]=o$. Thus we may assume that $\lambda<1$. Next note that

$$
\int_{0}^{1} f(t) \lambda\left\|f^{\prime}(t)\right\| d t=\lambda \mathrm{cm}[f]=o .
$$

This yields that

$$
\frac{\mathrm{cm}[f, \rho]}{(1-\lambda)}=\frac{1}{(1-\lambda)} \int_{0}^{1} f(t)(\rho(t)-\lambda)\left\|f^{\prime}(t)\right\| d t=\operatorname{cm}[f, \rho-\lambda] .
$$

Since $\lambda \leq 1 / \max \left[\left\|f^{\prime}\right\|\right]=\min [\rho]$, we have $\rho-\lambda \geq 0$. So Lemma 2.3 yields that

$$
\operatorname{cm}[f, \rho-\lambda] \in \operatorname{conv}[f],
$$

and completes the proof of (7). Next, to prove (8), note that if $\lambda<\min [\rho]$, then $\rho-\lambda>0$, in which case Lemma 2.3 again completes the proof. Finally, note that $\operatorname{supp}\left[\max \left[\left\|f^{\prime}\right\|\right]-\left\|f^{\prime}\right\|\right]$ is the closure of the set of points where $\rho \neq \min [\rho]$. Thus if $\lambda=\min [\rho]$ and we set $\widetilde{\rho}:=\rho-\lambda$, then $\operatorname{supp}\left[\max \left[\left\|f^{\prime}\right\|\right]-\left\|f^{\prime}\right\|\right]=\operatorname{supp}[\widetilde{\rho}]$. Then, once again by Lemma $2.3, \mathrm{~cm}[f, \widetilde{\rho}] \in \operatorname{relint} \operatorname{conv}[f]$, which would complete the proof of $(7)$.

\section{Spherical Loops with Prescribed Average and Length}

Here we show that for any point $p \in \mathbf{S}^{n-1}$ we may construct a closed curve with prescribed length $L$ and average $(1-\delta) p$ provided that $\delta / L$ is sufficiently small. This curve will begin and end at $p$ and can be constructed to smoothly join any given curve which passes through $p$. The proof of this result is divided into a sequence of lemmas presented below. The main idea is to start with a curve of length $L$ which is symmetric with respect to $p$ and shrink it continuously to $p$, while preserving its symmetry and length, until its average reaches the desired value. To achieve this we need to construct an appropriate family of contractions on the sphere which are defined with the aid of the functions given by the following lemma:

Lemma 3.1. For every interval $[0, b] \subset \mathbf{R}$ and $0<\epsilon<b$ there exists a continuous family of smooth functions $f_{t}:[0, b] \rightarrow[0, b], t \in[0,1)$, such that such that (i) $f_{0}(x)=$ $x$ for all $x \in[0, b]$, (ii) $f_{t}(x)=x$ for all $x \in[0,(1-t) \epsilon]$ (iii) $f_{t}$ is increasing (for each fixed $t$ ), and (iv) for any $x, y \in[0, b],\left|f_{t}(x)-f_{t}(y)\right|$ converges monotonically to 0 as $t$ approaches 1 , and is strictly decreasing if $x$ or $y$ lie in $[\epsilon, b]$. 
Proof. We construct $f_{t}$ by integrating an appropriate function (which we designate as $\left.f_{t}^{\prime}\right)$. Set $f_{t}(0):=0$. Then in order for $f_{t}$ to satisfy the properties enumerated above it is enough to have respectively (i) $f_{0}^{\prime} \equiv 1$ on $[0, b]$, (ii) $f_{t}^{\prime} \equiv 1$ on $[0,(1-t) \epsilon]$, (iii) $f_{t}^{\prime}>0$, and (iv) $\left|\int_{y}^{x} f_{t}^{\prime}(u) d u\right|$ converges monotonically to 0 and is strictly decreasing if $x$ or $y$ lie in $[\epsilon, b]$; in particular, to satisfy (iv) it is enough to require that $f_{t}^{\prime}(x)$ converge monotonically to 0 for each fixed $x>0$ and be strictly decreasing when $x \in[\epsilon, b]$.

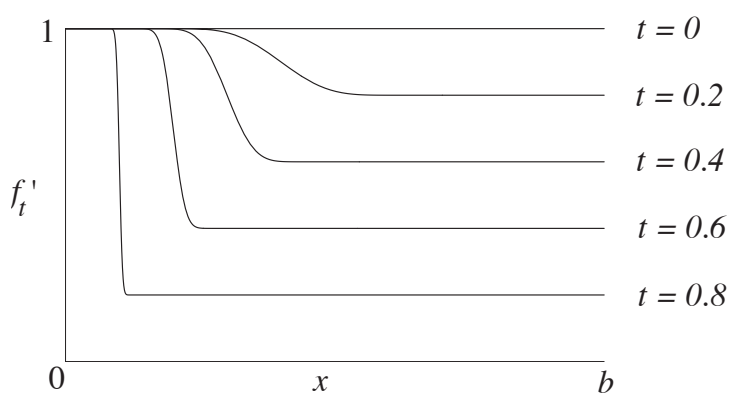

FiguRE 1

Figure 1 shows the graphs of some representatives from a family of functions which satisfy all the properties required of $f_{t}^{\prime}$. To construct this family explicitly we may set

$$
f_{t}^{\prime}(x):=1-t \psi_{t}(x)
$$

where $\psi_{t}$ is a continuous family of smooth nondecreasing step functions such that $\psi_{t} \equiv 0$ on $[0,(1-t) \epsilon]$, and $\psi_{t} \equiv 1$ on $\left[(1-t) \epsilon^{\prime}, b\right]$ for some $\epsilon<\epsilon^{\prime}<b$. In particular we may set

$$
\psi_{t}(x):=\frac{\int_{-\infty}^{x} \phi_{t}(u) d u}{\int_{-\infty}^{\infty} \phi_{t}(u) d u}
$$

where $\phi_{t}$ is the family of bump functions given by

$$
\phi_{t}(x):= \begin{cases}\exp \left(\frac{-1}{(x-(1-t) \epsilon)\left((1-t) \epsilon^{\prime}-x\right)}\right), & \text { if } \quad(1-t) \epsilon \leq x \leq(1-t) \epsilon^{\prime} \\ 0, & \text { otherwise. }\end{cases}
$$

For any pair of points $x, p \in \mathbf{S}^{n-1}$, the reflection $x^{\prime}$ of $x$ with respect to $p$ is defined as follows: if $x= \pm p$, then we set $x^{\prime}:=x$; otherwise we let $x^{\prime}$ be the point other than $x$ on the geodesic, or great circle, passing through $x$ and $p$ such that $\operatorname{dist}_{\mathbf{S}^{n-1}}\left(x^{\prime}, p\right)=\operatorname{dist}_{\mathbf{S}^{n-1}}(p, x)$. The reflection $X^{\prime}$ of any set $X \subset \mathbf{S}^{n-1}$ with respect to $p$ is defined by reflecting each of its points. If $X=X^{\prime}$ then we say that $X$ is symmetric with respect to $p$. A mapping $f: A \subset \mathbf{S}^{n-1} \rightarrow \mathbf{S}^{n-1}$ preserves symmetry with respect to $p$ provided that whenever $X \subset A$ is symmetric with respect to $p$, then $f(X)$ is symmetric with respect to $p$ as well. 
Lemma 3.2. Let $p \in \mathbf{S}^{n-1}$ and $H$ be a hemisphere centered at $p$. There exists a continuous family of smooth embeddings $\phi_{t}: H \rightarrow \mathbf{S}^{n-1}, t \in[0,1)$, which preserve symmetry with respect to $p$, and such that $\phi_{0}$ is the identity, each $\phi_{t}$ is the identity on some open neighborhood of $p, \phi_{t}$ converges to $p$ as $t$ approaches 1 , and for every pair of points $q, r \in H$ and $t>s$

$$
\operatorname{dist}_{\mathbf{S}^{n-1}}\left(\phi_{t}(q), \phi_{t}(r)\right) \leq \operatorname{dist}_{\mathbf{S}^{n-1}}\left(\phi_{s}(q), \phi_{s}(r)\right) .
$$

Finally, for any given open neighborhood $U$ of $p$, we may require that the above inequality be strict whenever $q$ or $r$ lie outside of $U$.

Proof. Let $f_{t}:[0, \pi / 2] \rightarrow[0, \pi / 2]$ be as in Lemma 3.1 where we choose $\epsilon$ so small that a ball of radius $\epsilon$ centered at $p$ is contained in $U$. Then we define $\phi_{t}(q)$ as the point on the geodesic segment connecting $q$ to $p$ such that

$$
\operatorname{dist}_{\mathbf{S}^{n-1}}\left(\phi_{t}(q), p\right)=f_{t}\left(\operatorname{dist}_{\mathbf{S}^{n-1}}(q, p)\right) .
$$

Since by Lemma $3.1 f_{t}$ is increasing for each fixed $t$ it follows that $\phi_{t}$ is an embedding. Further, with the exception of inequality (10) which we will verify below, it is clear that $\phi_{t}$ satisfies all the other desired properties as well. For instance, since each $f_{t}$ is the identity function close to 0 , it follows that each $\phi_{t}$ is the identity map close to $p$; and since $f_{t}$ converges to 0 on $(0, \pi / 2]$ it follows that $\phi_{t}$ converges to $p$.

In order to prove (10) we first abbreviate our notation as follows: for any $q \in \mathbf{S}^{n-1}$ let $\bar{q}:=\operatorname{dist}_{\mathbf{S}^{n-1}}(p, q)$ and $q_{t}:=\phi_{t}(q)$. Then by (11) we have

$$
\overline{q_{t}}=f_{t}(\bar{q}) .
$$

Next, applying the spherical law of cosines to the geodesic triangle $q_{t} p r_{t}$ we record that

$$
\cos \left(\operatorname{dist}_{\mathbf{S}^{n-1}}\left(q_{t}, r_{t}\right)\right)=\cos \overline{q_{t}} \cos \overline{r_{t}}+\sin \overline{q_{t}} \sin \overline{r_{t}} \cos \theta
$$

where $\theta$ is the angle of the geodesic triangle $q_{t} p r_{t}$ at $p$. Similarly,

$$
\cos \left(\operatorname{dist}_{\mathbf{S}^{n-1}}\left(q_{s}, r_{s}\right)\right)=\cos \overline{q_{s}} \cos \overline{r_{s}}+\sin \overline{q_{s}} \sin \overline{r_{s}} \cos \theta .
$$

Since the distance between any pairs of points of $H$ is at most $\pi$ and $\cos$ is decreasing on $[0, \pi]$, it follows that (10) holds if only we have

$$
\cos \left(\operatorname{dist}_{\mathbf{S}^{n-1}}\left(q_{t}, r_{t}\right)\right) \geq \cos \left(\operatorname{dist}_{\mathbf{S}^{n-1}}\left(q_{s}, r_{s}\right)\right)
$$

which is equivalent to

$$
\cos \overline{q_{t}} \cos \overline{r_{t}}-\cos \overline{q_{s}} \cos \overline{r_{s}} \geq\left(\sin \overline{q_{s}} \sin \overline{r_{s}}-\sin \overline{q_{t}} \sin \overline{r_{t}}\right) \cos \theta .
$$

Since by assumption $t>s$, it follows that $\overline{q_{t}} \leq \overline{q_{s}}$, because $\bar{q}_{t}=f_{t}(\bar{q})-f_{t}(0)$ is a nonincreasing function of $t$ by Lemma 3.1. Similarly $\overline{r_{s}} \geq \overline{r_{t}}$. So, since sin is increasing on $[0, \pi / 2]$, we have $\sin \overline{q_{s}} \sin \overline{r_{s}}-\sin \overline{q_{t}} \sin \overline{r_{t}} \geq 0$. Consequently (12) holds provided that

$$
\cos \overline{q_{t}} \cos \overline{r_{t}}-\cos \overline{q_{s}} \cos \overline{r_{s}} \geq \sin \overline{q_{s}} \sin \overline{r_{s}}-\sin \overline{q_{t}} \sin \overline{r_{t}}
$$

which may be rewritten as

$$
\cos \left(\overline{q_{t}}-\overline{r_{t}}\right) \geq \cos \left(\overline{q_{s}}-\overline{r_{s}}\right) .
$$


We may assume that $\bar{q} \geq \bar{r}$. Then $\overline{q_{t}} \geq \overline{r_{t}}$, since by Lemma 3.1, $f_{t}$ is increasing for each fixed $t$. Similarly $\overline{q_{s}} \geq \overline{r_{s}}$. So, since $\cos$ is decreasing on $[0, \pi / 2]$, the last displayed inequality holds provided that

$$
\overline{q_{t}}-\overline{r_{t}} \leq \overline{q_{s}}-\overline{r_{s}} .
$$

This is indeed the case by part (iv) of Lemma 3.1. Thus proof of (10) is complete. It remains to show that the inequality in (10) is strict whenever $q$ or $r$ do not lie in $U$. Suppose, for instance, that $q \notin U$. Then $\bar{q} \in[\epsilon, \pi / 2]$; therefore, by Lemma 3.1 , the last inequality displayed above is strict. This forces the inequality in (10) to be strict as well, as can be seen by going backwards through the chain of the last five inequalities displayed above, and observing that whenever one is strict, the subsequent one will also be strict.

Lemma 3.3. Let $f_{0} \in \operatorname{Imm}^{\alpha}\left([a, b], \mathbf{S}^{n-1}\right)$, and $f_{0}(a)=p=f_{0}(b)$. Suppose that $f_{0}([a, b])$ is contained in a hemisphere centered at $p$. Then there exists a continuous family of immersed curves $f_{t} \in \operatorname{Imm}^{\alpha}\left([a, b], \mathbf{S}^{n-1}\right), t \in[0,1)$, such that length $\left[f_{t}\right]=$ length $\left[f_{0}\right]$, and $f_{t}$ converges to $p$ as $t$ approaches 1 . Further we may require that for each $t$ there be an open neighborhood of the end points of $[a, b]$ where $f_{t}=f_{0}$, and $f_{t}$ be symmetric with respect to $p$ if $f_{0}$ has this symmetry.

Proof. We may suppose, for convenience, that $\left\|f_{0}^{\prime}\right\|=1$. Then length $\left[f_{0}\right]=b-a$. Let $\alpha_{t}:=\phi_{t} \circ f_{0}$, where $\phi_{t}$ is as in Lemma 3.2, and $U$ in Lemma 3.2 is chosen so small that it does not contain the entire image of $f_{0}$. Then $\alpha_{t} \in \operatorname{Imm}^{\alpha}\left([a, b], \mathbf{S}^{n-1}\right)$ is a continuous family which satisfies all the properties which are ascribed to $f_{t}$ above except that length $\left[\alpha_{t}\right]$ is strictly decreasing. In particular, for every $t \in[0,1)$ there exists a unique $s(t) \in[0,1)$ such that length $\left[\alpha_{s(t)}\right]=(1-t)(b-a)$. Thus, after a reindexing, we may suppose that length $\left[\alpha_{t}\right]=(1-t)(b-a)$. Further we may reparameterize so that

$$
\alpha_{t}:[a, b-t(b-a)] \rightarrow \mathbf{S}^{n-1},
$$

and $\alpha_{t}$ has unit speed. Next we define a family of complimentary curves $\beta_{t}$ : $[b-$ $t(b-a), b] \rightarrow \mathbf{S}^{n-1}$, by setting

$$
\beta_{t}(s):=\alpha_{1-t}(s-(1-t)(b-a)) .
$$

Now for $t \in[0,1 / 2]$ let $f_{t}:[a, b] \rightarrow \mathbf{S}^{n-1}$ be the curve which is obtained by joining $\alpha_{t}$ and $\beta_{t}$ in the natural way, i.e., set

$$
f_{t}(s):= \begin{cases}\alpha_{t}(s), & \text { if } s \in[a, b-t(b-a)] \\ \beta_{t}(s), & \text { if } s \in(b-t(b-a), b] .\end{cases}
$$

Then $f_{t}$ is a continuous family of $C^{\alpha}$-immersion. Further $f_{t}$ has constant speed 1 , so length $\left[f_{t}\right]=(b-a)=\operatorname{length}\left[f_{0}\right]$ as desired. Furthermore, note that $f_{1 / 2}$ double covers the trace of $\alpha_{1 / 2}$, since $\alpha_{1 / 2}$ and $\beta_{1 / 2}$ have identical traces. So we have shown that we may continuously deform $f_{0}$, while maintaining its length, symmetry and other required properties, until it double covers a curve of half the length of $f_{0}$. Similarly, we may iterate this procedure to define $f_{t}$, for $t \in[1 / 2,3 / 4]$, by starting with $f_{1 / 2}$ and end with a double covering of a curve which has half the length of $f_{1 / 2}$ 
or a quarter of the length of $f_{0}$. Thus we may define $f_{t}$ for all $t \in[0,1)$. This yields the desired family because whenever $t=\sum_{i=1}^{n} 1 / 2^{i}$ the trace of $f_{t}$ coincides with that of a curve whose length, and therefore distance from $p$, becomes arbitrarily small as $n$ gets large.

We are now ready to prove the main result of this section:

Proposition 3.4. Let $p \in \mathbf{S}^{n-1}, U \subset \mathbf{S}^{n-1}$ be an open neighborhood of $p$, and $\ell$, $c>0$. There exists $\bar{\delta}>0$ such that for any $0<\delta \leq \bar{\delta}$ and $[a, b] \subset \mathbf{R}$ with $(b-a) \geq \ell$ one may find an immersed curve $f:[a, b] \rightarrow U$ of constant speed $c$ such that

$$
\text { ave }[f]=(1-\delta) p
$$

$f(a)=p=f(b)$, and $f^{\prime}(a)$ is parallel to $f^{\prime}(b)$. Furthermore, if $C \subset \mathbf{S}^{n-1}$ is the image of any $\mathcal{C}^{\alpha \geq 1}$ embedded curve passing through $p$, then we may require that $f$ admit a $\mathcal{C}^{\alpha}$ extension $\tilde{f}:[a-\epsilon, b+\epsilon] \rightarrow \mathbf{S}^{n-1}$, for some $\epsilon>0$, such that

$$
\widetilde{f}([a-\epsilon, a] \cup[b, b+\epsilon]) \subset C .
$$

and $\widetilde{f}^{\prime}(a)$ is parallel to the direction of $C$ at $p$.
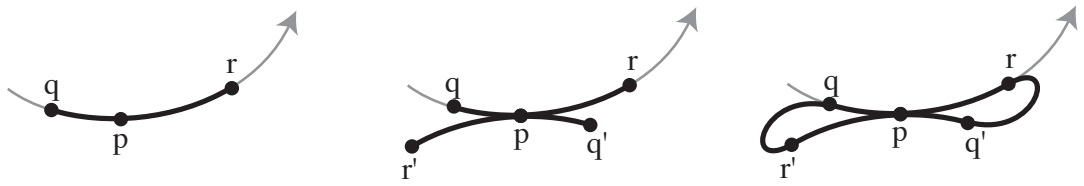

FiguRE 2

Proof. First we describe how to obtain $\bar{\delta}$. Consider a small segment of $C$ with end points $q$ and $r$ which contains $p$ in its interior and is contained in $U$, see Figure 2. We assume that $q$ precedes $p$ with respect to the direction of $C$. Let $q^{\prime} r^{\prime}$ be the reflection of the segment $q r$ with respect to $p$. Choosing $q$ and $r$ sufficiently close to $p$, we can make sure that the segment $q^{\prime} r^{\prime}$ lies in $U$. Further note that $q^{\prime}$ and $r$ can be made arbitrarily close provided that the length of the segment $q r$ is sufficiently small. In particular, we may choose the length, say $\lambda$, of the segment $q r$ so small, that the distance between $r$ and $q^{\prime}$ is less than $(\ell c-2 \lambda) / 2$ and the (shortest) geodesic segment connecting $r$ and $q^{\prime}$ lies in $U$. Then, since by assumption $(b-a) \geq \ell, r$ and $q^{\prime}$ may be joined to each other by a smooth segment $r q^{\prime}$ of length $((b-a) c-2 \lambda) / 2$ in $U$ which meets the segments $q r$ and $q^{\prime} r^{\prime}$ smoothly. Reflecting $q r^{\prime}$ with respect to $p$, we obtain a segment $q^{\prime} r$ which joins the other ends of the segments $q r$ and $q^{\prime} r^{\prime}$. Thus we obtain a smooth closed curve of length $(b-a) c$ which we may parametrize by $f:[a, b] \rightarrow \mathbf{S}^{n-1}$ of constant speed $c$ so that $f(a)=p=f(b)$ and $f$ passes through the points $p, r, q^{\prime}, p, r^{\prime}, q, p$ in that order. By construction, $f$ has the desired extension $\widetilde{f}$. Further, by symmetry, ave $[f]=k p$. For some $0<k<1$. We set $\bar{\delta}:=1-k$. Then $\|$ ave $[f]-p \|=\bar{\delta}$. Now recall that, by Lemma 3.3, we may continuously shrink $f$ towards $p$ while preserving its symmetry and length. 
This makes $\|$ ave $[f]-p \|$ arbitrarily small in a continuous way. In particular we may require that $\|$ ave $[f]-p \|=\delta$ for any given $0<\delta \leq \bar{\delta}$ which completes the proof.

\section{Constant Speed Approximations for Spherical Curves}

We now use the main results of the previous sections to show that any spherical curve $f$ may be approximated by another spherical curve with the same average as $f$ and speed equal to any given constant not smaller than the maximum speed of $f$. This is achieved by adding a number of small loops to the image of $f$. To find the locations where these loops should to be added, and the required length for each loop, we need to recall a pair of results from classical convexity theory.

Lemma 4.1 (Steinitz, [10]). Let $X \subset \mathbf{R}^{n}$, and $p \in \operatorname{int} \operatorname{conv} X$. Then there exist $2 n$ (or fewer) points $x_{i} \in X$ such that $p \in \operatorname{int} \operatorname{conv}\left\{x_{1}, \ldots, x_{2 n}\right\}$.

Lemma 4.2 (Kalman, [7]). Let $P \subset \mathbf{R}^{n}$ be a convex polytope with vertices $v_{i}$, $i=1, \ldots, k$. Then there exists continuous functions $\phi_{i}: P \rightarrow \mathbf{R}$ such that $\phi_{i} \geq 0$, $\sum_{i=1}^{k} \phi_{i}=1$, and, for every $x \in P, x=\sum_{i=1}^{k} \phi_{i}(x) v_{i}$.

Note 4.3. For any given $x \in \operatorname{int} P$, we may choose the functions $\phi_{i}$ in Lemma 4.2 so that $\phi_{i}(x)>0$. This is achieved by replacing $\phi_{i}$ with $\phi_{i}+\theta_{i}$ where $\theta_{i}$ are appropriately chosen functions with $\sum_{i=1}^{k} \theta_{i}=0$. More explicitly, note that for any $x \in P$ there exists $j \in\{1, \ldots, k\}$ such that $\phi_{j}(x)>0$, because $\sum_{i=1}^{k} \phi_{i}=1$. In particular, there exists $\epsilon>0$ such that $\phi_{j}(x)-\epsilon>0$. So we may set $\theta_{j}:=-\epsilon$ and, for $i \neq j$, set $\theta_{i}:=\epsilon /(k-1)$.

We say that a curve $f \in \operatorname{Imm}^{\alpha}\left([a, b], \mathbf{R}^{n}\right)$ is closed if $f^{(i)}(a)=f^{(i)}(b)$, for $0 \leq i \leq$ $\alpha$. Then we may write $f \in \operatorname{Imm}^{\alpha}\left(\Gamma, \mathbf{R}^{n}\right)$, where $\Gamma=\mathbf{R} /(b-a) \mathbf{Z}$. The main result of this section is:

Theorem 4.4. Let $f \in \operatorname{Imm}^{\alpha}\left([a, b], \mathbf{S}^{n-1}\right), U \subset \mathbf{S}^{n-1}$ be an open neighborhood of $f([a, b])$, and $c \geq \max \left[\left\|f^{\prime}\right\|\right]$. Suppose that int $\operatorname{conv}[f] \neq \emptyset$, and, furthermore, either $c>\max \left[\left\|f^{\prime}\right\|\right]$, or there exists no proper support hyperplane of $f([a, b])$ which contains $f\left(\operatorname{supp}\left[\max \left[\left\|f^{\prime}\right\|\right]-\left\|f^{\prime}\right\|\right]\right)$. Then there exists $\widetilde{f} \in \operatorname{Imm}^{\alpha}\left([a, b], \mathbf{S}^{n-1}\right)$ with constant speed c such that $\widetilde{f}([a, b]) \subset U, \int_{a}^{b} f(x) d x=\int_{a}^{b} \widetilde{f}(x) d x, \widetilde{f}(a)=f(a), \widetilde{f}(b)=$ $f(b)$, and for every $\eta>0$ there exists $\widetilde{\eta}>0$ such that $\widetilde{f}([a, a+\widetilde{\eta}]) \subset f([a, a+\eta])$ and $\tilde{f}([b-\widetilde{\eta}, b]) \subset f([b-\eta, b])$. In particular, if $f$ is closed then so is $\widetilde{f}$.

Proof. We construct $\tilde{f}$ by adding loops to $f$ at a number of points which are selected as follows. First note that if $\left\|f^{\prime}\right\|=c$, then we may simply set $\widetilde{f}:=f$ and we will be done. Otherwise $\lambda:=$ ave $\left\|f^{\prime}\right\| / c<1$, since by assumption $c \geq \max \left[\left\|f^{\prime}\right\|\right]$. Consequently, since by assumption relint conv $[f]=\operatorname{int} \operatorname{conv}[f]$, Proposition 2.4 yields that

$$
\frac{\operatorname{ave}[f]-\lambda \operatorname{cm}[f]}{(1-\lambda)} \in \operatorname{int} \operatorname{conv}[f]
$$


Since int $\operatorname{conv}[f]$ is a nonempty open set, we may choose $\bar{\delta}$ so small that for every $0 \leq \delta \leq \bar{\delta}$

$$
q(\delta):=\frac{\operatorname{ave}[f]-\lambda \operatorname{cm}[f]}{(1-\lambda)(1-\delta)} \in \operatorname{int} \operatorname{conv}[f]
$$

Now by Steinitz theorem (Lemma 4.1) there are $k \leq 2 n$ point $t_{i} \in[a, b]$ such that

$$
q(\delta) \in \operatorname{int} \operatorname{conv}\left\{f\left(t_{1}\right), \ldots, f\left(t_{k}\right)\right\} .
$$

Choosing $k$ as small as possible, we may assume that each $f\left(t_{i}\right)$ is a vertex of the polytope $\operatorname{conv}\left\{f\left(t_{1}\right), \ldots, f\left(t_{k}\right)\right\}$. These are the points to which we will add our loops. Next we will describe how to determine the size of each loop. Note that

$$
q(\delta)=\sum_{i=1}^{k} \phi_{i}(\delta) f\left(t_{i}\right),
$$

where $\phi_{i}(\delta):=\phi_{i}(q(\delta))$ are the coefficients given by Lemma 4.2. In particular $\phi_{i}:[0, \bar{\delta}] \rightarrow \mathbf{R}$ are continuous. Further recall (see Note 4.3 ) that we may assume that $\phi_{i}(0)>0$. Thus, choosing $\bar{\delta}>0$ sufficiently small, we can make sure that $\phi_{i}(\delta)>0$ for all $\delta \in[0, \bar{\delta}]$. In particular, letting $\underline{\phi_{i}}$ be the minimum of $\phi_{i}$ on $[0, \bar{\delta}]$, we may record that

$$
\phi_{i}(\delta) \geq \underline{\phi_{i}}>0
$$

These $\phi_{i}(\delta)$, for a sufficiently small $\delta>0$, are used to determine the size of the loops as we describe below.

In order to define $\tilde{f}$ we divide $[a, b]$ into $2 k+1$ subintervals. To this end first let $t_{0}:=a, t_{k+1}:=b$, and, for $i=1, \ldots, k+1$, let

$$
L_{i}:=\int_{t_{i-1}}^{t_{i}}\left\|f^{\prime}(t)\right\| d t
$$

be the distance between $f\left(t_{i-1}\right)$ and $f\left(t_{i}\right)$ along $f$. Next let $L=\sum_{i=1}^{k+1} L_{i}$ be the total length of $f$ and define $x_{j}, j=0, \ldots, 2 k+1$, recursively as follows depending on whether $j=0, j$ is odd $(j=2 i-1, i=1, \ldots, k+1)$, or $j$ is even $(j=2 i$, $i=1, \ldots, k)$ :

$$
x_{0}:=a, \quad x_{2 i-1}:=x_{2 i-2}+\frac{L_{i}}{L} \lambda(b-a), \quad x_{2 i}:=x_{2 i-1}+(1-\lambda)(b-a) \phi_{i}(\delta) .
$$

Note that

$$
x_{2 i}-x_{2 i-1} \geq(1-\lambda)(b-a) \underline{\phi_{i}}>0 .
$$

Let $\bar{\delta}_{i}$ be the upperbounds obtained from Proposition 3.4 by setting $p:=f\left(t_{i}\right)$, $U:=U, c:=c$, and $\ell:=(1-\lambda)(b-a) \underline{\phi_{i}}$. Now choose $\delta \in(0, \bar{\delta})$ so small that $\delta \leq \min \bar{\delta}_{i}$. Then we may define $\tilde{f}:[a, b] \rightarrow \mathbf{S}^{n-1}$ as the curve with constant speed

$$
\left\|\tilde{f}^{\prime}(t)\right\|=\frac{L}{\lambda(b-a)}=\frac{\text { ave }\left\|f^{\prime}\right\|}{\lambda}=c,
$$

which maps the (odd) intervals $\left[x_{2 i-2}, x_{2 i-1}\right], i=1, \ldots, k+1$, to $f\left(\left[t_{i-1}, t_{i}\right]\right)$, and maps the (even) intervals $\left[x_{2 i-1}, x_{2 i}\right], i=1, \ldots, k$, to the loops given by Proposition 
3.4 , with $p:=f\left(t_{i}\right), U:=U, c:=c,[a, b]:=\left[x_{2 i-1}, x_{2 i}\right]$ and $C$ a segment of the image of $f$ near $f\left(t_{i}\right)$.

We claim that $\widetilde{f}$ is the desired curve. To see this first note that Proposition 3.4 implies

$$
\frac{1}{b-a} \int_{x_{2 i-1}}^{x_{2 i}} \widetilde{f}(t) d t=\frac{x_{2 i}-x_{2 i-1}}{b-a} \text { ave }\left[\left.\widetilde{f}\right|_{\left[x_{2 i-1}, x_{2 i}\right]}\right]=(1-\lambda) \phi_{i}(\delta)(1-\delta) f\left(t_{i}\right),
$$

for $i=1, \ldots, k$. So, by $(13)$,

$$
\frac{1}{b-a} \sum_{i=1}^{k} \int_{x_{2 i-1}}^{x_{2 i}} \tilde{f}(t) d t=(1-\lambda)(1-\delta) q(\delta)=\operatorname{ave}[f]-\lambda \operatorname{cm}[f],
$$

Secondly note that by (3)

$$
\text { ave }\left[\left.\widetilde{f}\right|_{\left[x_{2 i-2}, x_{2 i-1}\right]}\right]=\mathrm{cm}\left[\left.\widetilde{f}\right|_{\left[x_{2 i-2}, x_{2 i-1}\right]}\right]=\mathrm{cm}\left[\left.f\right|_{\left[t_{i-1}, t_{i}\right]}\right] .
$$

So it follows that

$$
\frac{1}{b-a} \sum_{i=1}^{k+1} \int_{x_{2 i-2}}^{x_{2 i-1}} \widetilde{f}(t) d t=\lambda \sum_{i=1}^{k+1} \frac{L_{i}}{L} \mathrm{~cm}\left[\left.f\right|_{\left[t_{i-1}, t_{i}\right]}\right]=\lambda \mathrm{cm}[f],
$$

because the center of mass of a curve is the weighted average of the centers of mass of its subsegments. Now adding both sides of (15) and (16) yields that

$$
\operatorname{ave}[\tilde{f}]=\operatorname{ave}[f]
$$

as desired.

Note 4.5. In the statement of the previous proposition we may also require that $\widetilde{f}$ be $C^{0}$-close to $f$. This follows immediately by applying the last result to small subsegments of $f$.

\section{Proof of Theorem 1.1}

First we will record the following simple observation which will allow us to assume that the initial curve $f_{0}$ in Theorem 1.1 has unit speed.

Lemma 5.1. Let $f \in C^{1}\left([a, b], \mathbf{R}^{n}\right)$, and $\phi:[a, b] \rightarrow[a, b]$ be $C^{1}$. Then

$$
\|f \circ \phi\|_{1} \leq\|f\|_{1}\left(1+\|\phi\|_{1}\right) .
$$

Proof. Definition of $\|\cdot\|_{1}$, which is given in (1), immediately implies that

$$
\|f \circ \phi\|_{1} \leq \max \left\{\|f\|_{0},\|f\|_{1}\|\phi\|_{1}\right\} \leq\|f\|_{0}+\|f\|_{1}\|\phi\|_{1},
$$

which completes the proof since $\|f\|_{0} \leq\|f\|_{1}$.

Next we prove a pair of lemmas which are applications of Thom's transversality theorem. These results allow us to assume that the curvature of the initial curve $f_{0}$ in Theorem 1.1 behaves nicely enough so that its tantrix satisfies the hypothesis of the main result of the previous section, Theorem 4.4. In particular, the first lemma below allows us to assume that the set of points where the curvature of $f_{0}$ is strictly less than its maximum does not lie in a hyperplane. 
Lemma 5.2. Let $f \in \operatorname{Imm}^{\alpha \geq 2}\left([a, b], \mathbf{R}^{n}\right), \kappa$ be the curvature of $f$, and $c \geq \max [\kappa]$. Suppose $\kappa \not \equiv c$. Then for every $\epsilon>0$ there exists $\bar{f} \in \operatorname{Imm}^{\alpha}\left([a, b], \mathbf{R}^{n}\right)$ with curvature $\bar{\kappa}$ such that $\max [\bar{\kappa}] \leq c,\|f-\bar{f}\|_{2} \leq \epsilon, \bar{f}=f$ on an open neighborhood of the end points of $[a, b]$, and $\bar{f}(\operatorname{supp}[\max [\bar{\kappa}]-\bar{\kappa}])$ does not lie in any hyperplane.

Proof. By Thom's transversality theorem [1], the first $n$ derivatives of a $\mathcal{C}^{n}$-dense set of mappings $\tilde{f} \in \mathcal{C}^{n}\left([a, b], \mathbf{R}^{n}\right)$ are linearly independent everywhere except at a finite set of points. We will quickly verify this fact in the next paragraph. Then it follows that for a dense set of curves $\widetilde{f} \in \operatorname{Imm}^{n}\left([a, b], \mathbf{R}^{n}\right)$ there exists no open set $U \subset[a, b]$ such that $\widetilde{f}(U)$ lies in a hyperplane $H$, for then all the derivatives of $\widetilde{f}$ at points of $U$ would also have to lie in the $(n-1)$-dimensional subspace of $\mathbf{R}^{n}$ parallel to $H$. Let $\widetilde{f}_{i}$ be a sequence of these totally non-planar curves such that $\left\|f-\widetilde{f}_{i}\right\|_{2} \rightarrow 0$. We glue $\widetilde{f}_{i}$ to $f$ as follows. Let $U \subset[a, b]$ be an open set with closure $\bar{U} \subset(a, b)$ such that $\kappa<c$ on $\bar{U}$ (this is possible since $\kappa \not \equiv c$ by assumption). Let $\phi:[a, b] \rightarrow \mathbf{R}$ be a $\mathcal{C}^{\infty}$ function such that $\operatorname{supp} \phi \subset U, 0 \leq \phi \leq 1$, and $\phi \equiv 1$ on an open set $V \subset U$. Set $f_{i}:=(1-\phi) f+\phi \widetilde{f}_{i}$. Then, since $\phi$ is fixed, an elementary computation quickly shows that $\left\|f_{i}-f\right\|_{2} \rightarrow 0$. Thus $\left\|\kappa_{i}-\kappa\right\| \rightarrow 0$, where $\kappa_{i}$ and $\kappa$ are curvatures of $f_{i}$ and $f$ respectively. Consequently, for $i$ sufficiently large $\kappa_{i}<c$ on $U$. Since $\kappa_{i}=\kappa$ on $\Gamma-U$, it then follows that $\max \left[\kappa_{i}\right] \leq c$, when $i$ is large enough. But $f_{i}=\tilde{f}_{i}$ on $V$, thus $f_{i}(V)$ does not lie in any hyperplane. So when $i$ is sufficiently large, $\bar{f}:=f_{i}$ is the desired curve, since $V \subset \operatorname{supp}\left[\max \left[\kappa_{i}\right]-\kappa_{i}\right]$.

It remains only to verify the existence of the sequence $\widetilde{f}_{i}$, i.e., we need to show that the set of mappings $\widetilde{f}_{i} \in \mathcal{C}^{n}\left([a, b], \mathbf{R}^{n}\right)$ whose first $n$ derivatives are linearly independent everywhere except at a finite set of points are $\mathcal{C}^{n}$-dense. To this end let

$$
J^{n}\left([a, b], \mathbf{R}^{n}\right)=[a, b] \times \mathbf{R}^{n(n+1)}
$$

be the space of the $n$-jets of mappings $f:[a, b] \rightarrow \mathbf{R}^{n}$. We recall that, for any $f \in \mathcal{C}^{n}\left([a, b], \mathbf{R}^{n}\right)$, the corresponding $n$-jet $j^{n} f:[a, b] \rightarrow J^{n}\left([a, b], \mathbf{R}^{n}\right)$ is given by

$$
j^{n} f(t)=\left(t, f(t), f^{\prime}(t), \ldots, f^{(n)}(t)\right) .
$$

Let $A \subset J^{n}\left([a, b], \mathbf{R}^{n}\right)$ consist of those elements whose last $n$-coordinates are not linearly independent. The set of last $n$ elements of $A$ may be identified with the set of $n \times n$ matrices of rank less than $n$. These matrices form a stratified space of dimension $n^{2}-1$, e.g. see [1, p. 16]. So $\operatorname{dim}(A)=1+n+\left(n^{2}-1\right)$ which yields that $\operatorname{codim}(A)=1$. By Thom's transversality theorem, for a $\mathcal{C}^{n}$-dense set of maps $\tilde{f} \in \mathcal{C}^{n}\left([a, b], \mathbf{R}^{n}\right), j^{n} \tilde{f}$ is transversal to $A$. Since $\operatorname{dim}([a, b])=\operatorname{codim}(A)$, it follows that $j^{n} \widetilde{f}$ may intersect $A$ only at a discrete set, which, since $[a, b]$ is compact, must be finite. So the last $n$ derivatives of $\widetilde{f}$ are linearly dependent only at a finite set of points.

The next lemma allows us to assume that the curvature of the initial curve $f_{0}$ in Theorem 1.1 does not vanish. 
Lemma 5.3. Let $f \in \operatorname{Imm}^{\alpha \geq 2}\left([a, b], \mathbf{R}^{n}\right), \kappa$ be the curvature of $f, c \geq \max [\kappa]$, and $c>0$. Then for any $\epsilon>0$, there exists $\widetilde{f} \in \operatorname{Imm}^{\alpha}\left([a, b], \mathbf{R}^{n}\right)$ with curvature $\widetilde{\kappa}>0$ such that $|f-\widetilde{f}|_{\alpha} \leq \epsilon, \max [\widetilde{\kappa}] \leq c, \widetilde{f}$ is tangent to $f$ along any finite number of prescribed points, and $\widetilde{f}=f$ on any given open set $U \subset[a, b]$ where $\kappa>0$ on the closure $\bar{U}$.

Proof. By Thom's transversality theorem, there exists a dense set of curves $\widetilde{f}_{i} \in$ $\mathcal{C}^{\alpha}\left([a, b], \mathbf{R}^{n}\right)$ which have nonvanishing curvature. As in the proof of the previous lemma, we demonstrate this fact in the end. First, we use the existence of $\tilde{f}_{i}$ to show that we may assume that the curvature of $f$ does not vanish at the set of the prescribed points. To see this let $p \in[a, b]$ be one of these points, and suppose that $\kappa(p)=0$. Then $p \notin \bar{U}$. Further note that since $\left\|f-\widetilde{f}_{i}\right\|_{1} \rightarrow 0$, we may assume, after composing $\widetilde{f}_{i}$ with a sequence of rigid motions converging to the identity, that $\widetilde{f}_{i}(p)=f(p)$, and the tangent line of $\widetilde{f}_{i}$ at $p$ is parallel to the tangent line of $f$ at $p$. Let $V \subset[a, b]$ be an open neighborhood of $p$ such that $V$ is disjoint from $\bar{U}$, does not contain any other prescribed point, and $\kappa<c$ on $\bar{V}$. Let $\phi:[a, b] \rightarrow \mathbf{R}$ be a $\mathcal{C}^{\infty}$ function with support in $V$ such that $0 \leq \phi \leq 1$ and $\phi=1$ on an open neighborhood of $p$. Now set $\bar{f}_{i}:=\phi \widetilde{f}_{i}+(1-\phi) f$. Then $\overline{\bar{f}}_{i}$ differs from $f$ only on $V$, and $\left\|\bar{f}_{i}-f\right\|_{\alpha} \rightarrow 0$. In particular, since $\left\|\bar{f}_{i}-f\right\|_{2} \rightarrow 0$, curvature $\bar{\kappa}_{i}$ of $\bar{f}_{i}$ converges to $\kappa$ which is strictly less than $\max [\kappa]$ on $\bar{V}$. So it follows that $\max \left[\bar{\kappa}_{i}\right] \leq \max [\kappa]$. Further, by construction, $\bar{\kappa}_{i}(p)=\widetilde{\kappa}_{i}(p)>0, \bar{f}_{i}(p)=\widetilde{f}_{i}(p)=f(p)$, and since $\bar{f}_{i}$ coincides with $\widetilde{f}_{i}$ over an open neighborhood of $p$, the tangent line of $\bar{f}_{i}$ at $p$ is parallel to that of $f$. Thus After replacing $f$ with $\bar{f}_{i}$, for $i$ sufficiently large, we may assume that $\kappa(p) \neq 0$.

After performing the above procedure for each of the prescribed points $p \notin \bar{U}$, we may assume that $\kappa$ does not vanish at the prescribed points. In particular, there exists an open neighborhood $W \subset[a, b]$ disjoint from $\bar{U}$ and the set of prescribed points, which contains all the points of $[a, b]$ where $\kappa$ vanishes. Let $W_{1}$ be a connected component of $W$. Then $\kappa>0$ near the end points of $W_{1}$. Let $\psi:[a, b] \rightarrow \mathbf{R}$ be a $\mathcal{C}^{\infty}$ function with support in $W_{1}$ such that $0 \leq \psi \leq 1$ and $\kappa>0$ on the closure of the set of points of $W_{1}$ where $\psi<1$, e.g., $\psi=1$ everywhere except on a sufficiently small neighborhood of the end points of $W_{1}$. Then after replacing $f$ with $\widetilde{f}_{i} \psi+(1-\psi) f$, for $i$ sufficiently large, we may assume that $\kappa>0$ on $W_{1}$. Repeating this procedure for each component of $W$ yields that we may assume $\kappa>0$ everywhere.

Finally it remains to verify that there exists a dense set of curves $\widetilde{f}_{i} \in \mathcal{C}^{\alpha}\left([a, b], \mathbf{R}^{n}\right)$ which have nonvanishing curvature. To this end note that the curvature of a curve vanishes if and only if its first two derivatives are linearly dependent. The argument will now be similar to that at the end of the proof of the previous lemma. Consider the jet space $J^{2}\left([a, b], \mathbf{R}^{n}\right)=[a, b] \times \mathbf{R}^{3 n}$, and let $A \subset J^{2}\left([a, b], \mathbf{R}^{n}\right)$ consist of those elements whose last two components are not linearly independent. Then the set of the last two elements of $A$ may be identified with the set of $n \times 2$ matrices of rank less than 2, which has dimension $n+1$ [1, p. 16]. Thus $\operatorname{dim}(A)=1+n+(n+1)$, and so $\operatorname{codim}(A)=n-1$. By Thom's transversality theorem, there exists a dense 
set of curves $\widetilde{f}_{i} \in \mathcal{C}^{\alpha}\left([a, b], \mathbf{R}^{n}\right)$ whose jet $j^{2} \widetilde{f}_{i}$ is transversal to $A$. Since $n \geq 3$, $\operatorname{dim}([a, b])<\operatorname{codim}(A)$. So it follows that $j^{2} \widetilde{f}_{i}$ is disjoint from $A$, and thus has positive curvature.

With the aid of the previous two approximation results, we may now prove a local version of our main theorem:

Proposition 5.4. Let $f \in \operatorname{Imm}^{\alpha \geq 2}\left([a, b], \mathbf{R}^{n}\right)$ and $\kappa$ be the curvature of $f$. For any $c \geq \max [\kappa]$ and $\epsilon>0$, there exists $\widetilde{f} \in \operatorname{Imm}^{\alpha}\left([a, b], \mathbf{R}^{n}\right)$ with constant curvature $c$ such that $\|f-\widetilde{f}\|_{1} \leq \epsilon$, and $\tilde{f}$ is tangent to $f$ at $a, b$ and any other finite number of prescribed points. If $f$ is closed then so is $\widetilde{f}$, and if $\kappa=c$ on an open neighborhood of the end points of $[a, b]$, then we may require that $\widetilde{f}=f$ in some open neighborhood of $a$ and $b$.

Proof. First we show that it is enough to consider the case where $\left\|f^{\prime}\right\|=1$. To see this note that, after a dilation of $\mathbf{R}^{n}$, we may assume that length $[f]=b-a$. Then it is easy to construct a diffeomorphism $\theta:[a, b] \rightarrow[a, b]$ such that the reparameterized curve $f \circ \theta$ has unit speed; specifically, we may set $\theta:=s^{-1}(t)$, where $s(t):=$ $a+\int_{a}^{t}\left\|f^{\prime}(u)\right\| d u$. Next note that if the proposition holds for unit speed curves, then we may apply it to $f \circ \theta$ and obtain a curve $\widetilde{f \circ \theta}$, with the desired curvature properties, such that $\|f \circ \theta-\widetilde{f \circ \theta}\|_{1} \leq \epsilon /\left(1+\left\|\theta^{-1}\right\|_{1}\right)$. Now set $\widetilde{f}:=\widetilde{f \circ \theta} \circ \theta^{-1}$. Then by Lemma 5.1

$$
\|f-\widetilde{f}\|_{1}=\left\|(f \circ \theta-\widetilde{f \circ \theta}) \circ \theta^{-1}\right\|_{1} \leq\|f \circ \theta-\widetilde{f \circ \theta}\|_{1}\left(1+\left\|\theta^{-1}\right\|_{1}\right) \leq \epsilon .
$$

So $\tilde{f}$ is the desired curve, since curvature is invariant under reparameterization, and, furthermore, if $f \circ \theta=\widetilde{f \circ \theta}$ on an open neighborhood of the end points of $[a, b]$, then we also have $f=\widetilde{f}$ over some (smaller) open neighborhood of $a$ and $b$ as well.

If $\left\|f^{\prime}\right\|=1$, which we may assume from now on, then the tantrix $T=f^{\prime}$ and consequently $\kappa=\left\|T^{\prime}\right\|$. Further note that if $c=0$, then we may simply set $\widetilde{f}:=f$ and we will be done. Otherwise we may assume, by Lemma 5.3, that $\kappa>0$. Then $T \in \operatorname{Imm}^{\alpha-1}\left([a, b], \mathbf{S}^{n-1}\right)$. Divide $[a, b]$ into $k$ subsegments $\left[a_{i-1}, a_{i}\right]$ where $a_{0}:=a$, $a_{k}:=b$, and $\left\{a_{0}, \ldots, a_{k}\right\}$ includes all the prescribed points where we would like to have $\tilde{f}$ tangent to $f$. Choosing $k$ sufficiently large, we may assume that each $\left|a_{i-1}-a_{i}\right|$ is small enough so that

$$
\text { length }\left[\left.T\right|_{\left[a_{i-1}, a_{i}\right]}\right]<\epsilon
$$

for all $i$. Then $T\left(\left[a_{i-1}, a_{i}\right]\right)$ will be contained in an open ball $U_{i} \subset \mathbf{S}^{n-1}$ of (spherical) radius $\epsilon / 2$. Let $\bar{f}$ be the $\mathcal{C}^{1}$ perturbation of $f$ obtained by applying Lemma 5.2 to $f$ restricted to each interval $\left[a_{i-1}, a_{i}\right]$. Since $\bar{f}$ can be made arbitrarily $\mathcal{C}^{1}$-close to $f$, we may assume, after a reparameterization, that it has unit speed, and its tantrix satisfies

$$
\bar{T}\left(\left[a_{i-1}, a_{i}\right]\right) \subset U_{i}
$$

Let $\bar{T}_{i}$ be the restriction of $\bar{T}$ to each interval $\left[a_{i-1}, a_{i}\right]$ and note that, by construction, $\bar{T}_{i}\left(\operatorname{supp}\left[\max \left[\left\|\bar{T}_{i}^{\prime}\right\|\right]-\left\|\bar{T}_{i}^{\prime}\right\|\right]\right)$ does not lie in any hyperplane, unless $\bar{T}_{i}$ has 
constant speed $c$. Thus we may apply Theorem 4.4 to each $\bar{T}_{i}$ to obtain for each $i$ a mapping $\widetilde{T}_{i} \in \operatorname{Imm}^{\alpha-1}\left(\left[a_{i-1}, a_{i}\right], \mathbf{S}^{n-1}\right)$ with constant speed $c$. Define $\widetilde{T}$ by setting it equal to $\widetilde{T}_{i}$ on each $\left[a_{i-1}, a_{i}\right]$. Then $\widetilde{T} \in \operatorname{Imm}^{\alpha-1}\left([a, b], \mathbf{S}^{n-1}\right),\left\|\widetilde{T}^{\prime}\right\|=c$, $\|T-\widetilde{T}\|_{0} \leq \epsilon, \widetilde{T}\left(a_{i}\right)=T\left(a_{i}\right)$, and $\int_{a_{i-1}}^{a_{i}} \widetilde{T}(t) d t=\int_{a_{i-1}}^{a_{i}} T(t) d t$. Now, for $t \in[a, b]$, set

$$
\widetilde{f}(t):=f(a)+\int_{a}^{t} \widetilde{T}(u) d u .
$$

Then $\tilde{f} \in \operatorname{Imm}^{\alpha}\left([a, b], \mathbf{R}^{n}\right),\|f-\widetilde{f}\|_{1} \leq \epsilon$, and $\widetilde{f}$ is tangent to $f$ at $a_{i}$.

Finally recall that, by Theorem 4.4, the trace of $T$ coincides with that of $\widetilde{T}$ near the end points of $[a, b]$. Thus, if $T$ has constant speed $c$ near the end points of $[a, b]$, then $\widetilde{T}=T$ near the endpoints of $[a, b]$ which in turn implies that $\widetilde{f}=f$ on that region as well.

Our main result now quickly follows from the previous proposition:

Proof of Theorem 1.1. Let $X \subset \Gamma$ be the given compact set where $\kappa_{0}=c$ on an open neighborhood of $X$. If $X=\emptyset$, then Proposition 5.4 completes the proof. Otherwise let $U$ be an open neighborhood of $X$ where $\kappa_{0}=c$. Choosing $U$ sufficiently close to $X$, we may assume that $\kappa_{0}=c$ near the end points of each of the segments of $\Gamma-U$. Let $f_{1}:=f_{0}$ on $\mathrm{U}$, and set $f_{1}:=\widetilde{f}$ on $\Gamma-U$, where $\widetilde{f}$ is obtained from Proposition 5.4 applied to each segment of $\Gamma-U$ by setting $f=f_{0}$. Then $f_{1} \in \operatorname{Imm}^{\alpha}\left(\Gamma, \mathbf{R}^{n}\right)$ and, since immersions and embeddings are open in $\mathcal{C}^{1}\left(\Gamma, \mathbf{R}^{n}\right), f_{t}:=(1-t) f_{0}+t f_{1}$, $t \in[0,1]$, yields the desired homotopy, provided that we choose the $\epsilon$ in Proposition 5.4 sufficiently small. Specifically, note that

$$
\left\|f_{t}-f_{0}\right\|_{1}=t\left\|f_{1}-f_{0}\right\|_{1} \leq\left\|f_{1}-f_{0}\right\|_{1} \leq \epsilon .
$$

So $\epsilon$ needs to be chosen so small that whenever $\left\|f_{0}-g\right\|_{1} \leq \epsilon$ then $g$ is an immersion, and furthermore $g$ is an embedding when $f_{0}$ is an embedding.

\section{APPENDIX}

Here we check that the lower bound for the constant $c$ in Theorem 1.1 is optimal, i.e., if $f \in \operatorname{Imm}^{2}\left(\Gamma, \mathbf{R}^{n}\right)$ is any immersed curve and $\widetilde{f} \in \operatorname{Imm}^{2}\left(\Gamma, \mathbf{R}^{n}\right)$ has constant curvature $c$ which is strictly less than the maximum curvature of $f$, then $\tilde{f}$ cannot be arbitrarily $\mathcal{C}^{1}$-close to $f$. To this end we require the following basic lemma. Recall that a partition $P=\left\{t_{0}, \ldots, t_{k}\right\}$ of an interval $[a, b] \subset \mathbf{R}$, is a finite set of points such that $a=t_{0}<\cdots<t_{k}=b$. The length of a curve $f \in \mathcal{C}^{0}\left([a, b], \mathbf{R}^{n}\right)$ with respect to a partition $P$ is defined as

$$
\operatorname{length}[f ; P]:=\sum_{i=1}^{k}\left\|f\left(t_{j-1}\right)-f\left(t_{j}\right)\right\|,
$$

and the length of $f$ is given by

$$
\text { length }[f]:=\sup _{P \in \text { Partition }[a, b]} \operatorname{length}[f ; P],
$$


where Partition $[a, b]$ denotes the set of all partitions of $[a, b]$. As is well-known, when $f$ is $\mathcal{C}^{1}$ this definition for length coincides with the analytical one given by (2). If length $[f]$ is finite, then we say that $f$ is rectifiable.

Lemma 5.5. Let $f \in \mathcal{C}^{0}\left([a, b], \mathbf{R}^{n}\right)$ be a rectifiable curve and $f_{i} \in \mathcal{C}^{0}\left([a, b], \mathbf{R}^{n}\right)$ be any sequence of rectifiable curves which converges to $f$ with respect to the $\mathcal{C}^{0}$-norm. Then

$$
\liminf \text { length }\left[f_{i}\right] \geq \operatorname{length}[f] .
$$

Proof. Let $P=\left\{t_{0}, \ldots, t_{k}\right\}$ be a partition of $[a, b]$. Note that $\left.f_{i}\right|_{\left[t_{j-1}, t_{j}\right]}$ is a connected curve whose end points lie within spheres of radii $\left\|f_{i}-f\right\|_{0}$ centered at $f\left(t_{j-1}\right)$ and $f\left(t_{j}\right)$. Thus

$$
\text { length }\left[\left.f_{i}\right|_{\left[t_{j-1}, t_{j}\right]}\right] \geq\left\|f\left(t_{j-1}\right)-f\left(t_{j}\right)\right\|-2\left\|f_{i}-f\right\|_{0} .
$$

Summing both sides of the above inequality over $j$, we obtain

$$
\text { length }\left[f_{i}\right] \geq \operatorname{length}[f ; P]-2 n\left\|f_{i}-f\right\|_{0} .
$$

Since by assumption, $\left\|f_{i}-f\right\|_{0} \rightarrow 0$ as $i \rightarrow \infty$ we then conclude that

$$
\liminf \text { length }\left[f_{i}\right] \geq \operatorname{length}[f ; P] .
$$

This completes the proof since the partition $P$ was arbitrary.

Now we are ready to prove the main result of this section:

Proposition 5.6. Let $f \in \operatorname{Imm}^{2}\left(\Gamma, \mathbf{R}^{n}\right)$ and $\widetilde{f}_{i} \in \operatorname{Imm}^{2}\left(\Gamma, \mathbf{R}^{n}\right)$ be a sequence such that each $\widetilde{f}_{i}$ has constant curvature $c$ and $\widetilde{f}_{i}$ converges to $f$ with respect to the $\mathcal{C}^{1}$ norm. Then $c \geq \max [\kappa]$, where $\kappa$ is the curvature of $f$.

Proof. After reparameterizing $f$ and $\tilde{f}_{i}$ by arclength, and passing to a subsequence of $\widetilde{f}_{i}$, we may assume that $\widetilde{f}_{i}$ and $f$ have unit speed. Then the tantrices $\widetilde{T}_{i}=\widetilde{f}_{i}^{\prime}$ have constant speed $c$ and converge to $T=f^{\prime}$ with respect to the $\mathcal{C}^{0}$-norm. By Lemma 5.5 , for any fixed $t_{0} \in \Gamma$ and $\epsilon>0$, we have

$$
\liminf \text { length }\left[\left.\widetilde{T}_{i}\right|_{\left[t_{0}-\epsilon, t_{0}+\epsilon\right]}\right] \geq \operatorname{length}\left[\left.T\right|_{\left[t_{0}-\epsilon, t_{0}+\epsilon\right]}\right]
$$

Thus, since $T$ and $\widetilde{T}_{i}$ are $\mathcal{C}^{1}$, we may write

$$
\int_{t_{0}-\epsilon}^{t_{0}+\epsilon}\left\|\widetilde{T}_{j}^{\prime}(t)\right\| d t \geq \int_{t_{0}-\epsilon}^{t_{0}+\epsilon}\left\|T^{\prime}(t)\right\| d t-\delta_{i}
$$

for all $j \geq N(i)>i$, where $\delta_{i} \rightarrow 0$ as $i \rightarrow \infty$. This in turn may be rewritten as

$$
c \geq \frac{1}{2 \epsilon} \int_{t_{0}-\epsilon}^{t_{0}+\epsilon}\left\|T^{\prime}(t)\right\| d t-\frac{\delta_{i}}{2 \epsilon},
$$

since $\left\|\widetilde{T}_{i}^{\prime}\right\|=c$ by assumption. Taking the limit as $i \rightarrow \infty$ now yields that

$$
c \geq \frac{1}{2 \epsilon} \int_{t_{0}-\epsilon}^{t_{0}+\epsilon}\left\|T^{\prime}(t)\right\| d t,
$$


for any $t_{0} \in \Gamma$ and $\epsilon>0$. Thus, taking the limit as $\epsilon \rightarrow 0$, we have

$$
c \geq\left\|T^{\prime}\left(t_{0}\right)\right\|=\kappa\left(t_{0}\right)
$$

for any $t_{0} \in \Gamma$. In other words $c \geq \max [\kappa]$ as claimed.

\section{ACKNOWLEDGMENTS}

The author is grateful to David Spring for his detailed reading of earlier drafts of this work, and suggestions which led to improving its exposition.

\section{REFERENCES}

[1] Y. Eliashberg and N. Mishachev. Introduction to the h-principle, volume 48 of Graduate Studies in Mathematics. American Mathematical Society, Providence, RI, 2002.

[2] C. Engelhardt. Raumkurven konstanter Krümmung, insbesondere Gewundene Kreise. Ph.D. Thesis, 2005.

[3] E. A. Feldman. Deformations of closed space curves. J. Differential Geometry, 2:67-75, 1968.

[4] M. Ghomi and M. Kossowski. $h$-principles for hypersurfaces with prescribed principle curvatures. Tran. Amer. Math. Soc., To appear.

[5] H. Gluck and L.-H. Pan. Embedding and knotting of positive curvature surfaces in 3-space. Topology, 37(4):851-873, 1998.

[6] M. Gromov. Partial differential relations. Springer-Verlag, Berlin, 1986.

[7] J. A. Kalman. Continuity and convexity of projections and barycentric coordinates in convex polyhedra. Pacific J. Math., 11:1017-1022, 1961.

[8] R. Koch and C. Engelhardt. Closed space curves of constant curvature consisting of arcs of circular helices. J. Geom. Graph., 2(1):17-31, 1998.

[9] J. McAtee. Knots of constant curvature. arXiv:math.GT/0403089 v1, 2004.

[10] R. Schneider. Convex bodies: the Brunn-Minkowski theory. Cambridge University Press, Cambridge, 1993.

[11] D. Spring. Convex integration theory, volume 92 of Monographs in Mathematics. Birkhäuser Verlag, Basel, 1998. Solutions to the $h$-principle in geometry and topology.

School of Mathematics, Georgia Institute of Technology, Atlanta, GA 30332

E-mail address: ghomi@math.gatech.edu

$U R L$ : www . math. gatech. edu/ ghomi 